number may have had non cardiac chest pain. The CAD50 rate in the $\mathrm{ACP}, \mathrm{AP}$ and $\mathrm{AB}$ groups (50\% of total cases) was only $10 \%$ suggesting that CTA is largely being used to exclude significant $\mathrm{CAD}$ in low or intermediate risk groups. The rate of CAD 70 in group A was only 20\%. It is likely that figure is artificially low as, while waiting lists for CTA remain longer than for ICA, clinicians will request ICA as the first test if CAD70 felt likely. Better case selection for CTA could free up capacity for PTs with AA and A. Without this or substantial further increases in CTA capacity, the number of ICAs performed not leading to revascularisation is unlikely to change.

Conflict of Interest None

\section{Stable IHD/Prevention/Hypertension/Lipids}

\section{THE PREVALENCE OF CORONARY ARTERY DISEASE IN PATIENTS PRESENTING TO A RAPID ACCESS CHEST PAIN CLINIC WITH 'NON-ANGINAL CHEST PAIN'}

Will Chick, Anita Macnab. Wythenshawe Hospital, Manchester, UK

\subsection{6/heartjn-2021-BCS.178}

Introduction NICE define three key features of typical angina, and patients presenting with only one of these features are described as having 'non-anginal chest pain'. The latest NICE guidelines advise against routine testing for coronary artery disease (CAD) in this cohort. Despite this, the rapid access chest pain clinic at our institution have continued to investigate these patients. This study sought to establish the prevalence of CAD in this cohort, and evaluate the differences in cardiovascular risk factors between those with and without significant disease.

Methods Between 1st January 2017 and 1st December 2019, patients without prior history of cardiac disease presenting with non-anginal chest pains were referred for coronary computed tomography angiography (CCTA). Calcification scores were recorded using the Coronary Artery Calcium-Data and Reporting System (CAC-DRS) and luminal stenosis was recorded using the Coronary Artery Disease-Reporting and Data System (CAD-RADS). Patients unable to have luminal analysis due to calcium or high heart rates either had stress echocardiography (SE) or invasive coronary angiography (iCA). Patients were deemed as having significant CAD if they had any of the following: CAD-RADS score of 4 or more on CCTA, 4 segments of stress inducible ischaemia on SE or severe stenosis on iCA. Cardiovascular risk factors were then compared between those with and without CAD, to determine if there were any significant differences between the groups.

Results In total, 1078 patients presented with non-anginal chest pains. All patients had CAC-DRS scores and in 872 patients it was possible to record CAD-RADS scores (table 1). Of the 206 patients unable to have luminal analysis, 199 had SE and 7 had iCA. In total, 143 patients (13.3\%) were found to have significant $\mathrm{CAD}$ on imaging. In total, 34 of these patients had revascularisation ( 9 patients had bypass grafting and 25 patients had percutaneous coronary intervention). Table 2 shows that patients with significant $\mathrm{CAD}$ were more likely to be hypertensive $(p=0.004)$, older $(p<0.001)$ and have a higher Qrisk2 score $(\mathrm{p}<0.001)$.

Conclusion Following current NICE guidelines for 'non-anginal chest pain' may mean a considerable proportion of patients
Abstract 181 Table 1 The CAD-RADS scores of patients with a CCTA allowing luminal analysis

\begin{tabular}{ll}
\hline CAD-RADS score & Number of patients \\
\hline 0 & 261 \\
1 & 194 \\
2 & 190 \\
3 & 126 \\
$4 \mathrm{~A}$ or $4 \mathrm{~B}$ & 90 \\
5 & 11 \\
\hline
\end{tabular}

Abstract 181 Table 2 The differences in cardiovascular risk factors in those with and without significant CAD

\begin{tabular}{lllll}
\hline $\begin{array}{l}\text { Cardiovascular risk } \\
\text { factor }\end{array}$ & $\begin{array}{l}\text { All } \\
(\mathbf{n}=1078)\end{array}$ & $\begin{array}{l}\text { Patients without } \\
\text { significant CAD } \\
(\mathbf{n}=935)\end{array}$ & $\begin{array}{l}\text { Patients with } \\
\text { significant CAD } \\
(\mathbf{n}=143)\end{array}$ & $\begin{array}{l}\mathbf{P} \\
\text { value }\end{array}$ \\
\hline Age (years), mean (SD) & $60(10.4)$ & $59(10.5)$ & $63(9.1)$ & $<0.001$ \\
Qrisk2, median (IQR) & 14.2 & $13.3(7.8-21.2)$ & $19.6(12.4-26.2)$ & $<0.001$ \\
& $(8.1-22.5)$ & & & \\
Diabetes, $\mathrm{n}(\%)$ & $167(15)$ & $139(15)$ & $28(20)$ & 0.171 \\
Hypertension, $\mathrm{n}(\%)$ & $416(39)$ & $345(37)$ & $71(50)$ & 0.004 \\
Family History, $\mathrm{n}(\%)$ & $429(40)$ & $370(40)$ & $59(41)$ & 0.714 \\
Hypercholesterolaemia, & $168(16)$ & $147(16)$ & $21(15)$ & 0.806 \\
$\mathrm{n}(\%)$ & & & & \\
\hline
\end{tabular}

with underlying CAD are missed, including those with severe lesions requiring revascularisation. Our analysis suggests that age, Qrisk2 score and a history of hypertension are statistically significant predictors of significant CAD in this population.

Conflict of Interest None

\section{TISSUE DOPPLER E' VELOCITY AND E/E' PREDICT 19- YEAR CARDIOVASCULAR MORTALITY IN HYPERTENSION}

${ }^{1}$ Anenta Ramakrishnan, ${ }^{2}$ Anoop Shah, ${ }^{1}$ Simon Thom, ${ }^{3}$ Andrew Sharp, ${ }^{1}$ Darrel Francis,

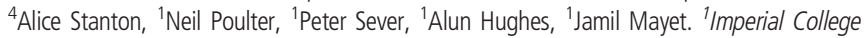
London, London, UK; ${ }^{2}$ London School of Hygiene and Tropical Medicine; ${ }^{3}$ University of Exeter; ${ }^{4}$ Royal College of Surgeons in Ireland

\subsection{6/heartjnl-2021-BCS.179}

Background We have previously shown that tissue Doppler assessments of left ventricular (LV) diastolic function predict cardiac events in a hypertensive population over a period of 4 years. These out-performed traditional echocardiographic measures in a well-treated hypertensive population.

Purpose We aimed to test whether tissue Doppler assessment of LV diastolic function would predict cardiovascular (CV) mortality in the Hypertension Associated Cardiovascular Disease sub-study of the Anglo-Scandinavian Cardiac Outcomes Trial (ASCOT).

Methods ASCOT was a multicentre randomised trial with a $2 \times 2$ factorial design. Inclusion criteria for the study included hypertension and three other CV risk factors, including male sex and age over 55. Protocols, including for echocardiography, have been detailed previously.This study comprised the 